\title{
Issues associated with polarisation independence in silicon photonics
}

\author{
Graham T. Reed ${ }^{\mathrm{a}}$, Goran Z Mashanovicha, William R Headley ${ }^{\mathrm{a}}$, Branislav Timotijevic ${ }^{\mathrm{a}}$, \\ Frederic Y Gardes ${ }^{\mathrm{a}}$, Seong Phun Chan ${ }^{\mathrm{a}}$, Peter Waugh ${ }^{\mathrm{a}}$, Neil G Emerson ${ }^{\mathrm{a}}$, Ching Eng Png ${ }^{\mathrm{b}}$, \\ Mario J Paniccia ${ }^{\mathrm{c}}$ IEEE Senior Member, Ansheng Liu ${ }^{\mathrm{c}}$, Dani Hak ${ }^{\mathrm{d}}$, \\ and Vittorio M. N. Passaro ${ }^{\mathrm{e}}$ IEEE Senior Member \\ ${ }^{a}$ Advanced Technology Institute, School of Electronics and Physical Sciences, \\ University of Surrey, Guildford, Surrey, GU2 7XH, UK \\ Tel.+44(0)1483 689122,Fax+44(0)1483 689404,Email: g.reed@surrey.ac.uk \\ ${ }^{b}$ Now with The Institute of High Performance Computing, A*Star, 1 Science Park Road, \#01-01 \\ The Capricorn, Singapore Science Park II, Singapore 117528 \\ ${ }^{c}$ Intel Corporation, 2200 Mission College Blvd, SCI2-326, Santa Clara, CA, USA 95054 \\ ${ }^{d}$ Intel Corporation, 5 Office Building, S.B.I. Park Har Hotzvim, 91031, Jerusalem, Israel \\ ${ }^{e}$ Dipartimento di Elettrotecnica ed Elettronica, Politecnico di Bari, \\ Via E. Orabona 4, 70125 Bari, Italy
}

\begin{abstract}
Silicon Photonics is experiencing a dramatic increase in interest due to emerging applications areas and several high profile successes in device and technology development. Despite early work dating back to the mid 1980s, dramatic progress has been made in recent years. Whilst many approaches to research have been developed, the striking difference between the work of the early to mid 1990s, and more recent work, is that the latter has been associated with a trend to reduce the cross sectional dimensions of the waveguides that form the devices. The question arises therefore as to whether one should move to very small strip waveguides (silicon wires) of the order of $250 \mathrm{~nm}$ in height and a few hundred nanometres in width for improved device
\end{abstract}


performance but with little hope of polarisation independence, or to utilise slightly larger rib waveguides that offer more opportunity to control the polarisation dependence of the devices. In this paper we discuss devices suitable for one approach or the other and present designs associated both with strip and rib waveguides. In particular, we present designs of polarisation independent ring resonators with FSRs up to $12 \mathrm{~nm}$, we propose modulators for bandwidths in the $10 \mathrm{~s}$ of $\mathrm{GHz}$ regime, and we present grating based couplers for rib and strip waveguides, and/or for wafer scale testing, as well as a novel means of developing Bragg gratings via ion implantation.

Index Terms: Silicon-On-Insulator (SOI), rib waveguides, single mode condition, polarisation independence, optical modulators, Bragg gratings, grating couplers, ring resonators

\section{INTRODUCTION}

Silicon Photonics is a research field that is surprisingly mature in some senses, having been studied since the mid 1980s. In other ways, it is in its infancy, with some major advances being reported only very recently. The first waveguides were reported in the mid 1980s, in silicon on doped silicon [1], silicon on sapphire [2], silicon germanium [3], and Silicon on Insulator (SOI) [4,5]. The silicon on insulator platform, first reported in 1989, has by far, become the most popular of the four waveguide systems, and it is this platform that has formed the core of the work carried out by the Silicon Photonics Group at the University of Surrey, which was also established in 1989. The first results from the group were published in early 1991, demonstrating waveguides formed by the SIMOX process [6]. These early waveguides 
exhibited losses as high as $30 \mathrm{~dB} / \mathrm{cm}$, but within a year the same group had reported waveguides with a loss of less than $1 \mathrm{~dB} / \mathrm{cm}$, demonstrating the viability of the technology [7].

The first silicon based optical modulators were proposed in 1986, with modelling suggesting a $\pi$ radian phase shift could be achieved in a device less than a $1 \mathrm{~mm}$ long. The corresponding loss was less than $1 \mathrm{~dB}$ at $\lambda=1.3 \mu \mathrm{m}$ for both TE and TM polarisations [8]. However, the electrical power densities required to drive early modulators was very high, and it was not until 1993 that the Surrey group proposed a 3-terminal device that reduced power consumption by an order of magnitude $[9,10]$. Device variants had bandwidths of up to $20 \mathrm{MHz}$, and were based upon a waveguide with large cross sectional dimensions of the order of $6 \mu \mathrm{m}$, and a drive current of only $7 \mathrm{~mA}$ [11]. Nevertheless more recent work has proposed and/or demonstrated fast modulators in smaller waveguides, with drive currents below $1 \mathrm{~mA}$. Some of these devices will be discussed later, together with our other work on ring resonators, grating based devices and optical couplers. Firstly however, let us consider some fundamental issues associated with shrinking the waveguide cross sectional dimensions.

\section{FUNDAMENTAL WAVEGUIDE ISSUES}

Single-mode SOI rib waveguides with large cross section have been studied extensively by a number of researchers [12-17] to find single mode behaviour at the same time as low propagation loss. The majority of these photonic devices in SOI have been studied in waveguides that are multi-micron in cross sectional dimensions (of the order of $5 \mu \mathrm{m}$ ), to facilitate low-loss coupling to and from optical fibres. Soref et al. [13] first proposed a simple expression for these large ribs waveguides, related to their geometry to ensure that they satisfied the single-mode condition (SMC): 


$$
\frac{W}{H} \leq \alpha+\frac{r}{\sqrt{1-r^{2}}}, \text { for } 0.5 \leq \mathrm{r} \leq 1
$$

where $r$ is the ratio of slab height to overall rib height, $\mathrm{W} / H$ is the ratio of waveguide width to overall rib height, and $\alpha=0.3$. The analysis of the waveguides was limited to shallow etched ribs $(r>0.5)$, and hence deeply etched rib waveguides were not considered. Furthermore, the waveguide dimensions were assumed to be larger than the operating wavelength. Their analysis was based on the assumption that high order vertical modes (i.e. modes other than the fundamental mode) confined under the rib waveguides, were coupled to the outer slab region during propagation, therefore yielding high propagation losses for the higher order modes. Thus the waveguides behave as single mode waveguides, as all other modes are lost. Other authors have also considered the single mode for large waveguides, and produced similar expressions (e.g. [14], [15]). However, the current trend in silicon photonic circuits to move to smaller device dimensions for improved cost efficiency and device performance can come at some cost to other performance parameters, notably in the polarisation dependence of the circuits if they are not carefully designed. Furthermore, Soref's design equation (equation 1), cannot be applied to small and deeply etched rib waveguides. In order to maintain the consistency with other work $[13,15-$ 17], we have used the full-vectorial beam propagation method (BPM) [18] to analyse the deeply etched rib waveguide structure, mode propagation within it, and polarisation independence. Some results of the SMC condition have also been verified by the finite element method (FEM) [19]. For rib waveguides we have evaluated the single mode cut-off condition by determining when the first mode of higher order than the fundamental mode begins to propagate. Because the waveguides are relatively small, for high etch depths $(r<0.5)$, the single mode condition 
becomes dominated by boundary conditions, and hence the conditions for quasi-TE and quasiTM modes begin to diverge. We have also determined the difference between the effective indices of the fundamental mode as a function of waveguide width and etch depth (and hence parameter $r$ defined above), for a given waveguide height. The condition when the effective indices are equal is defined as the zero birefringence condition, and there are up to two such events for each waveguide etch depth. By determining these conditions we can plot a "zero birefringence locus" for each waveguide height [20].

The simulations were carried out for rib waveguides of silicon $\left(n_{\mathrm{g}}=3.477\right)$ on silica $\left(n_{\mathrm{s}}=1.444\right)$ and an upper cladding that is air $\left(n_{\mathrm{c}}=1\right)$, although it is a simple task to extend the work to an upper oxide cladding. SOI rib waveguides with an overall height in the range $H=1.00 \mu \mathrm{m}$, to $1.50 \mu \mathrm{m}$ were analysed at a wavelength of $1.55 \mu \mathrm{m}$. Both the single mode condition and the zero birefringence conditions can conveniently be plotted on the same curve to determine the waveguide parameters that allow both conditions to be satisfied simultaneously. For example, Figure 1 shows such plots for waveguide heights of $1.35 \mu \mathrm{m}$ (Figure 1(a)) and $1.5 \mu \mathrm{m}$ (Figure 1(b)). It can be seen from Figure 1 that for truly single mode behaviour, it is the quasi TM condition that is the limiting condition, because if this is satisfied, then the quasi TE condition is automatically satisfied. Consequently, for both single mode behaviour and polarisation independence, then the waveguide design should lie on the zero birefringence locus, below the quasi-TM single mode boundary, in the bottom right hand corner of figures 1(a) and 1(b). From this and other data [20], [21], we can extract design rules to aid the design of single mode rib waveguides, for waveguide heights in the range $H=1.00 \mu \mathrm{m}$ to $1.50 \mu \mathrm{m}$. 


$$
\begin{aligned}
& \frac{W}{H} \leq 0.05+\frac{(0.94+0.25 H) r}{\sqrt{1-r^{2}}} \text { for } r \leq 0.5 \text { and } 1.0 \leq H \leq 1.5 \\
& D_{\text {min }}=0.06 \times 10^{-6}+0.556 H
\end{aligned}
$$

Equation 2 defines the quasi-TM single mode boundary, and hence provides guidance on the geometric limitations to retain single mode behaviour, whilst equation 3 defines the minimum etch depth required to obtain polarisation independence.

\section{RING RESONATORS IN RIB WAVEGUIDES}

We can utilise the information of the previous section to design a polarisation independent ring resonator. Such a resonator requires polarisation independent waveguides for matching of the TE/TM phase shifts around the ring waveguides, but also requires polarisation independent directional couplers to transfer light to and from the ring. A directional coupler comprises two waveguides in close proximity such that the evanescent fields of the optical modes overlap, and light can transfer from one waveguide to the other. We have previously described how to design such a coupler [22], by allowing multiple passes of light from one of the waveguides in the directional coupler to the other. For example Figure 2(a) shows modelling of the optical power in the two arms of a directional coupler. For the purposes of the modelling, the left arm of the coupler was excited with an optical field. With propagation distance $z$, light transfers from the left waveguide to the right, and back again in a cyclic manner. The goal of the modelling is to

find a coupler length that yields the same degree of power transfer from the left waveguide to the right, for both polarisations. 
Figure 2(a) demonstrates that, power transfers in a shorter length for the TE polarisation than for TM. However for three transitions of the TM mode, and 5 transitions of the TE mode, the power in the left waveguide has transferred to the right waveguide, over a coupler length of approximately $500 \mu \mathrm{m}$, and hence for this fixed coupling length the device is polarisation independent. A ring resonator utilising this principle was fabricated, and the experimental characteristics are shown in Figure 2(b), which clearly demonstrates polarisation independent performance over a spectral width of three times the free spectral range (FSR). This device was based upon a waveguide height of $1.35 \mu \mathrm{m}$ and a rib width of $0.8 \mu \mathrm{m}$.

However, the resultant FSR is very small, due to the relatively large circumference of the ring resonator, which in turn is a direct result of the size of the waveguides. Consequently it could be argued that resonators based upon strip waveguides would be more useful as the ring circumference could be much smaller $(\sim 5 \mu \mathrm{m})$ leading to FSRs of the order of $10-20 \mathrm{~nm}$ (e.g. [23]). However, we can push the large ring resonators further either by simply shrinking the ring circumference, or by employing cascaded or multiple ring resonators. Let us first consider shrinking the ring circumference. The device resulting in the data of Figure 2(b) had a bend radius of $400 \mu \mathrm{m}$ and a total circumference of $3513 \mu \mathrm{m}$, resulting in an FSR of only $193 \mathrm{pm}$. We have also fabricated a series of other resonators with varying circumference, based upon identical waveguide dimensions. The results of these devices are summarised in Table 1. This table shows that if the bend radius is reduced to $25 \mu \mathrm{m}$, the FSR can be increased significantly to $815 \mathrm{pm}$. However, this is still very small for many applications. Consequently we can investigate both cascaded resonators and devices based upon serial coupling of multiple rings. 
We have carried out modelling of both such devices, based upon the experimental results summarised in Table 1. For example if we consider cascaded ring resonators based upon our

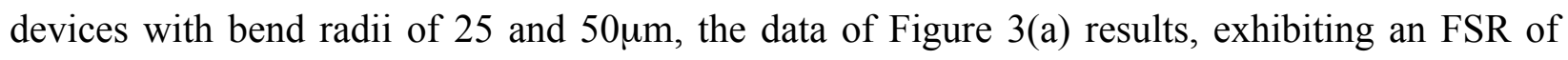
approximately $4.1 \mathrm{~nm}$. In such a configuration light propagates through the first resonator, straight section and finally through the second resonator towards the output drop port. If rings are different, resonant conditions are different and only wavelengths satisfying both of them will be present at the output. Therefore, the device acts as "and" function between two rings and the net transfer function (Figure 3(a)) can be approximated by multiplying responses of single racetrack resonators employing $310 \mu \mathrm{m}$-couplers and bend radii of $25 \mu \mathrm{m}$ and $50 \mu \mathrm{m}$ assuming the same coupling and propagation conditions as for single stage filters. Alternatively, a device with serially coupled rings of radii of 25 and $33.24 \mu \mathrm{m}$ and with the length of the coupler of $310 \mu \mathrm{m}$, exhibiting the so called Vernier effect, exhibit a much improved FSR of approximately $12 \mathrm{~nm}$. The value for the second radius has also been obtained by observing single-stage filter response for $25 \mu \mathrm{m}$ racetrack, assuming the same coupling conditions and taking into account Vernier condition $\mathrm{m} \times \mathrm{FSR}_{1}=\mathrm{n} \times \mathrm{FSR}_{2}$ [24]. By choosing these values for bend radii and the length of the coupler, the total FSR becomes 15 times bigger than the FSR for $25 \mu \mathrm{m}$-single ring, the spectrum of which is given in (Figure 3(b)), but it should be also noted that this design requires very careful matching of the coupling coefficients and, consequently, control of fabrication issues. This figure could be improved further by reducing the coupler length and hence the circumference by using MMI couplers. Nevertheless it is clear that FSRs are possible in polarisation independent resonators that approach the FSR performance of very small ring resonators that would almost certainly be highly polarisation dependent. 
It is worth considering one further improvement of the multiple ringed resonator structure. By serially coupling similar rings (rather than dissimilar rings for FSR improvement), we can vary the FWHM of the resonance, and hence change the effective Q factor of the device. For example Figure 4 shows experimental results of ring resonator devices with single, double and triple ringed devices, each with rings of the same size. The devices were based on the same waveguides as previously, and a directional coupler of $2010 \mu \mathrm{m}$, resulting in a full width half maximum (FWHM) of $27 \mathrm{pm}$ for a single ring device, $22 \mathrm{pm}$ for a dual ring device and $17 \mathrm{pm}$ for a triple ring device. Figure $4 \mathrm{~b}$ shows that the experimental results of a double ring resonator compares extremely well with the theoretical transfer function, demonstrating that we have excellent control of the design parameters of such devices.

\section{GRATING BASED DEVICES}

Gratings have numerous applications in optical circuits including filtering, coupling to/from circuits, and phase matching in directional couplers, and as Bragg reflectors. Since gratings are highly polarisation dependent devices, it is questionable whether there are applications in polarisation independent waveguides. However, if the grating can be made to respond only to one polarisation, then cascading two gratings can result in a device that is effectively polarisation independent. A Bragg reflector is an obvious example of such a device.

We have carried out grating work in silicon waveguides ranging in height from $1 \mu \mathrm{m}$ to $1.5 \mu \mathrm{m}$. Our earliest work dates back to 1998, and was based upon grating couplers for input/output coupling. Figure 5 shows grating couplers on the surface of $1 \mu \mathrm{m}$ waveguides. Figure 5a shows 
a conventional castellated grating that exhibited a measured out-coupling efficiency of $72 \%$ at a wavelength of $1.3 \mu \mathrm{m}$ [25], and Figure $5 \mathrm{~b}$ shows a blazed grating also on a $1 \mu \mathrm{m}$ waveguide that exhibited an increased out-coupling efficiency of $84 \%$ at a wavelength of $1.3 \mu \mathrm{m}$ [26]. Such gratings are useful for lab based coupling experiments, but may also have much wider application in wafer scale testing of silicon photonic circuits.

We are now working on similar gratings, as well as Bragg gratings, that can be formed by ion implantation of oxygen, or other species. The advantage of such an approach is that there is no physical etch of the waveguide surface, and planar surface retention is easier to process. Furthermore, they are more convenient for integration with other devices such as heaters to enable thermal tuning of the gratings, or perhaps with electronics. The development of gratings fabricated via ion implantation relies on a modification of the refractive index at the waveguide surface in a periodic manner. This can be achieved in silicon by ion implantation through a mask, and hence the design is critically dependent upon achieving good control of the surface implant. Thus modelling of the surface implant as well as the required subsequent annealing process is important. Grating depths up to $150 \mathrm{~nm}$ are being considered. Oxygen is a good choice for the implant, as the annealing process results in a definitive interface between the silicon dioxide resulting from the oxygen implant, and the remaining surface silicon, similar to work reported by Bussman et al [27]. Figure 6 shows the predicted implant profile of $30 \mathrm{keV}$ oxygen into silicon. Clearly the implant is not truly a surface implant, but the subsequent annealing process results in diffusion of oxygen into a near stoichiometric surface layer of $\mathrm{SiO}_{2}$. 
The preceding examples of grating structures are compatible with rib waveguides of the order of 1-2 $\mu \mathrm{m}$ in height. However, we have built upon our work on surface gratings to develop a coupler device for coupling into very small waveguides, such as silicon wires, for applications in which polarisation independence is less important. We have developed a device we have entitled the Dual Grating Assisted Directional Coupler (DGADC) [28].

A DGADC in SOI is shown in Fig. 7a [28]. A thick waveguide and two separation layers are fabricated in SiON for refractive index control over a broad range (1.45-2). The top layer is $5 \mu \mathrm{m}$ thick with refractive index close to the refractive index of optical fibre, resulting in an insertion loss $\leq 0.05 \mathrm{~dB}$ from the fibre to this first waveguide.

A fibre is butt coupled to the thick SiON waveguide and subsequently the light is coupled to a $\mathrm{Si}_{3} \mathrm{~N}_{4}$ waveguide using the first grating, and to the thin $(\approx 1 / 4 \mu \mathrm{m})$ SOI waveguide via the second grating. The silicon nitride waveguide is crucial for the operation of the device because it enables highly efficient coupling at both gratings, consequently forming an efficient DGADC. This waveguide bridges the gap between SiON and Si layers in both refractive index and thickness. The buried oxide layer serves as the lower cladding layer, for isolation from the substrate, hence removing any leakage loss towards the substrate. Figure $7 \mathrm{~b}$ shows the theoretical efficiency of the structure, achieved via modelling. The total efficiency is expressed as the product of the efficiencies of the first grating $\left(\eta_{1}\right)$ and the second grating $\left(\eta_{2}\right)$, as a function of the thickness of the $\mathrm{Si}_{3} \mathrm{~N}_{4}$. The efficiency is plotted for three different combinations of grating heights. The most important point to notice from this graph is that the theoretical coupling efficiency can exceed $90 \%$. We have yet to fabricate the optimum device, but we have recently reported preliminary 
results measured of a device with a surface waveguide height of $\sim 3.7 \mu \mathrm{m}$, which in turn means that the theoretical coupling is $60 \%[29]$, rather than $>90 \%$ in the optimized device. However, the experimentally determined efficiency was $55 \%$, very close to the theoretical value. This is already a competitive performance for coupling to such small waveguides, but perhaps more importantly, this result means that the DGADC remains amongst the most promising devices for very high efficiency coupling to very small waveguides. The measured bandwidth of $\sim 5 \mathrm{~nm}$ can be broaden significantly by chirping and by varying the duty cycle of the gratings.

\section{MODULATORS FOR RIB AND STRIP WAVEGUIDES}

Due to the fact that fast modulators in silicon must probably be realized via the plasma dispersion effect, modulators will always be faster and more efficient in a smaller waveguide as compared to a similar modulator in a larger waveguide. However, one of the fastest modulators reported to date has been fabricated in a rib waveguide [30]. Therefore both rib and strip waveguides are worthy of consideration.

In 2003/4, we reported simulation of a $p-i-n$ modulator based upon a rib waveguide with an overall waveguide height of approximately $1 \mu \mathrm{m}[31,32]$. The structure is shown in Figure 8a. The doping profile of the $n^{+}$regions was optimised to provide maximum modulation speed. This optimum doping profile in the side $n^{+}$regions was achieved through modelling of a series of different implantation steps such that the peak concentration at the surface of the $n^{+}$contact was approximately $10^{20} \mathrm{~cm}^{-3}$ and decreased to approximately $10^{16} \mathrm{~cm}^{-3}$ at the interface between the silicon and the buried oxide. The determination of this profile was achieved through the process simulation package, ATHENA from SILVACO [33]. The optimum profile predicted a current 
requirement to achieve a phase modulation of $\pi$ radians, $I_{\pi}$ of $0.7 \mathrm{~mA}$, as well as rise and fall times of $0.38 \mathrm{~ns}$ and $0.13 \mathrm{~ns}$ respectively, corresponding to a device bandwidth of the order of $1 \mathrm{GHz}$. We also showed that by overdriving, the rise and fall times of the modulator can be further improved to provide a bandwidth in excess of $5 \mathrm{GHz}$, an effect that has since been used by $\mathrm{Xu}$ et al [34] to overdrive a ring resonator modulator in a strip waveguide to achieve a data rate of $1.5 \mathrm{Gbit} / \mathrm{s}$.

We have recently reported modelling of a faster modulator suitable for width dimensions similar to those of a strip waveguide [35]. This device is shown in Figure 8b. It is fabricated in a waveguide that, whilst having dimensions approaching those of silicon wire dimensions, is technically still a rib waveguide, and hence some polarisation control is still possible. The device has an asymmetrical $p n$ structure where two slab regions are joined as a common cathode and two poly-silicon regions are joined as a common anode. Both $n^{+}$and $p^{+}$regions were modelled as highly doped regions with peak doping concentrations of $1 \times 10^{19} \mathrm{~cm}^{-3}$. The structure is based around an overall silicon thickness of $0.45 \mu \mathrm{m}$, etched rib waveguides $0.415 \mu \mathrm{m}$ wide with a slab thickness of $0.1 \mu \mathrm{m}$. These dimensions were chosen to approach polarisation independence operational regime. The silicon slab and the bottom part of the rib have an $n$-type background doping concentration of $4 \times 10^{17} \mathrm{~cm}^{-3}$ and the top part of the rib has a $p$-type background doping concentration of $2 \times 10^{17} \mathrm{~cm}^{-3}$. The $n^{+}$doped regions are situated on both sides of the wave guiding region, in the slab, $1.5 \mu \mathrm{m}$ from the centre of the waveguide. Furthermore the polysilicon $p^{+}$doped regions are situated on both sides of the top of the rib in order to reduce the losses resulting from the poly-silicon and aluminium contacts. 
The modulation mechanism was carrier depletion from the $p n$ junction. Carrier losses induced were minimised in our design. Modulation was proposed by including two devices in a Mach Zehnder interferometer, to operate in push-pull mode. In the 'off' state, both devices were reverse biased to $5 \mathrm{~V}$ such that no phase difference was induced between the arms of the Mach Zehnder. In the 'on' state, one arm was reverse biased to $10 \mathrm{~V}$ whilst the bias on the other was reduced to $0 \mathrm{~V}$, to induce a $\pi$ radian phase shift between the arms. The transient time of each device was characterised by ATLAS [33] in terms of carrier concentration against time. The carrier concentration profile for a reverse bias of 5 volts was taken as a reference. The change in carrier concentration compared to a reverse bias of 5 volts (against time) was converted to a change in refractive index profile (against time). This profile was then used in BeamPROP [18] simulation to determine the change in effective index, which was subsequently converted into a change in phase shift (with time).

The rise and fall times of the proposed device have both been calculated to be $7 \mathrm{ps}$ for a reverse a bias of 5 volts, predicting an intrinsic bandwidth of several 10 s of $\mathrm{GHz}$, although in practice large peak currents will probably reduce this somewhat. This modulator provides improved performance in terms of loss and bandwidth compared to the strip waveguide device of Barrios and Lipson [36]. For a more detailed overview of modulators in silicon photonics, see for example [37].

\section{CONCLUSION}

The current trend in silicon photonics is to move to small waveguide dimensions, resulting in increased difficulty in maintaining single mode operation whilst simultaneously designing for polarisation independence. However, it is possible to achieve such a design for small rib 
waveguides of the order of $1.0-1.5 \mu \mathrm{m}$ in height. We have provided guidelines to aid such design.

We have demonstrated a polarisation independent design in ring resonators where such behaviour is particularly important. The intrinsic FSR of such devices is small due to the relatively large circumference of ring resonators based upon rib waveguides, and it is for this reason that some other authors have moved to smaller devices fabricated in strip waveguides. However, we have demonstrated that it is possible to achieve respectable FSRs in polarisation independent rib waveguides, with a predicted FSR based on our existing results being as large as $12 \mathrm{~nm}$, and further improvement envisaged. We have also demonstrated additional control of the quality factor of such devices via serial cascading of multiple rings.

However, we also have an interest in strip waveguide work, and we have reported both grating based device designs and modulator designs in waveguides compatible with either strips or ribs. In particular the modulators offer the potential of multi tens of $\mathrm{GHz}$ modulation. In order to address the problem of coupling to very small waveguides we have designed and fabricated a DGADC for coupling to very small waveguides, promising coupling efficiencies up to $90 \%$, with $55 \%$ reported to date.

The question to whether to pursue polarisation independence however, remains complex. In particular it is related to the application in question. For example, it is likely that for optimum modulation speed, a strip waveguide based modulator placed directly in front of a laser would not be required to exhibit polarisation independence because the laser is inherently polarised. 
Alternatively, a modulator fed via an optical fibre is much more likely to be required to satisfy polarisation independent performance. Therefore we can envisage systems in which the performance of some devices is considerably more critical than others, and hence the question of whether to pursue polarisation independence must be viewed from the perspective of the application in question. Fortunately, the flexibility of silicon photonics means there is room for both approaches, and each approach has advantages in some application areas.

\section{ACKNOWLEDGEMENTS}

The authors are grateful to the following organisations for funding parts of this work:

The Engineering and Physical Sciences Research Council, UK.

Bookham Technology Inc., UK.

The Intel Corporation, USA.

Agilent Technologies, Singapore.

The University of Surrey. 


\section{REFERENCES}

[1] R A Soref, and J P Lorenzo, "All-silicon active and passive guided-wave components for $\lambda=1.3$ and 1.6 um," IEEE J. Quantum Electron., QE-22, 873-879. 1986.

[2] D J Albares, and R A Soref. "Silicon-on-sapphire waveguides," in Proc. SPIE: Integrated Optical Circuit Engineering IV, 704, 24 -25, 1987.

[3] B L Weiss, Z Yang, and F Namavar, "Wavelength dependent propagation loss characteristics of SiGe/Si planar waveguides,” Electron. Lett., 28, 2218-2220, 1992.

[4] E Cortesi, F Namavar, and R A Soref, "Novel silicon-on-insulator structures for silicon waveguides," in 1989 IEEE SOS/SOI Technology Conference (Cat. No.89CH2796-1), 3-5 Oct. 1989, Stateline, NV, USA: IEEE, 1989.

[5] F Namavar, E Cortesi, R A Soref, and P Sioshansi, "On the formation of thick and multiple layer SIMOX structures and their applications," in Ion Beam Processing of Advanced Electronic Materials Symposium, 25-27 April 1989, San Diego, CA, USA: Mater. Res. Soc., 1989.

[6] B L Weiss, G T Reed, S K Toh, R A Soref, and F Namavar, "Optical waveguides in SIMOX structures," IEEE Photonics Techol. Lett., $\underline{3}$, 19-21, 1991.

[7] A Rickman, G T Reed, B L Weiss, F Namavar, "Low-loss planar optical waveguides fabricated in SIMOX material," IEEE Photonics Techol. Lett., 4, 633-635, 1992.

[8] R A Soref, and B R Bennett, "Kramers-Kronig analysis of electro-optical switching in silicon," in Proc.SPIE: Integrated Optical Circuit Engineering IV, 16-17 Sept. 1986, Cambridge, MA, USA, 704, 32-37.

[9] C K Tang, G T Reed, A J Walton, and A G Rickman, "Simulation of a low loss optical modulator for fabrication in SIMOX material," in Silicon-Based Optoelectronic Materials Symposium, 12-14 April 1993, San Francisco, CA, USA: Mater. Res. Soc. 1993.

[10] C K Tang, G T Reed, A J Walton, and A G Rickman, "Low-loss, single-mode, optical phase modulator in SIMOX material," J. Lightwave Technol., 12, 1394-1400, 1994.

[11] C K Tang and G T Reed, "Highly efficient optical phase modulator in SOI waveguides," Electron. Lett., 31, 451-452, 1995.

[12] G T Reed and A P Knights, Silicon Photonics: An Introduction, ISBN 0-470-87034-6 (John Wiley, West Sussex, 2004). 
[13] R A Soref, J Schmidtchen and K Petermann, "Large single-mode rib waveguides in Ge-Si and Si-on-SiO 2 ," IEEE J. Quantum Electron., 27, 1971-1974, 1991.

[14] S P Pogossian, L Vescan, and A Vonsovici, "The single-mode condition for semiconductor rib waveguides with large cross section," J. Lightwave Technol., 16, 1851-1853, 1998.

[15] O Powell, "Single-mode condition for silicon rib waveguides," J. Lightwave Technol., 20, 1851-1855, 2002.

[16] L Vivien, S Laval, B Dumont, S Lardenois, A Koster, and E Cassan, "Polarizationindependent single-mode rib waveguides on silicon-on-insulator for telecommunication wavelengths," Opt. Commun., 210, 43-49, 2002.

[17] J Lousteau, D Furniss, A Seddon, T M Benson, A Vukovic and P Sewell, "The single-mode condition for silicon-on-insulator optical rib waveguides with large cross section," J. Lightwave Technol., 22, 1923-1929, 2004.

[18] BeamPROP by RSoft Design Group, Inc., 200 Executive Group Blvd. Ossining, NY 19562 . [19] FEMLAB by COMSOL, Inc., 1 New England Executive Park Suite, 350 Burlington, MA 01803.

[20] S P Chan, C E Png, S T Lim, V M N Passaro, and G T Reed, "Single mode and polarisation independent SOI waveguides with small cross section”, J. Lightwave Technol.,23, 1573-1582, 2005.

[21] S P Chan, V M N Passaro and G T Reed, "Single mode condition and zero birefringence in small SOI waveguides", Electron. Lett., 41, 528-529, 2005.

[22] W R Headley, G T Reed, M Paniccia, A Liu, and S Howe, 'Polarization-independent optical

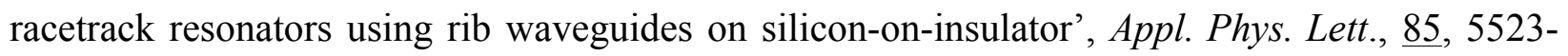
5525, 2004.

[23] R Baets, P Dumon, W Bogaerts, D Thourhout, D Taillaert, B Luyssaert, P Bienstman, V Wiaux, S Beckx, and J Wouters, "Silicon-on-insulator platform for WDM components," Proceedings of the 17th Annual Meeting of the IEEE, 2, 741-742, 2004.

[24] Y H Ja, "A Vernier fiber double-ring resonator with a 3x3 fiber coupler and degenerate twowave mixing," IEEE Photon. Technol. Lett., 4, 1479-1485, 1995.

[25] T W Ang, G T Reed, A Vonsovici, A G R Evans, P R Routley, M R Josey, "Effects of grating heights on highly efficient Unibond SOI waveguide grating couplers," IEEE Photon. Technol. Lett., 12, 59-61, 2000. 
[26] T W Ang, G T Reed, A Vonsovici, A G R Evans, P R Routley, and M R Josey, "Highly efficient Unibond silicon-on-insulator blazed grating couplers," Appl. Phys. Lett., 77, 42144216, 2000.

[27] U Bussmann, P F L Hemment, A K Robinson, and V V Starkov, “Oxygen implantation through patterned masks: a method for forming insulated device islands while maintaining a planar wafer surface," Nucl. Instr. Meth., B55, 856-859, 1991.

[28] G Z Masanovic, V M N Passaro, and G T Reed, "Dual grating-assisted directional coupling between fibres and thin semiconductor waveguides," IEEE Photon. Technol. Lett., 15, 13951397, 2003.

[29] G Z Masanovic, G T Reed, W Headley, B Timotijevic, V M N Passaro, R Atta, G Ensell, and A G R Evans, "A high efficiency input/output coupler for small silicon photonic devices," Opt. Express, 13, 7374-7379, 2005.

[30] L Liao, D Samara-Rubio, M Morse, A Liu, D Hodge, D Rubin, U D Keil, and T Franck, “High speed silicon Mach-Zehnder modulator," Opt. Express, 13, 3129-3135, 2005.

[31] C E Png, G T Reed R M Atta, G J Ensell, and A G R Evans, "Development of small silicon modulators in silicon-on-insulator (SOI)," Proc. SPIE,. 4997, 190-197, 2003.

[32] C E Png, S P Chan, S T Lim, and G T Reed, “Optical phase modulators for MHz and GHz modulation in silicon-On-insulator (SOI)," J. Lightwave Technol., 22, 1573-1583, 2004.

[33] Silvaco Internationnal, 4701 Patrick Henry drive,Bldg 1, Santa Clara, CA 94054

[34] Q Xu, B Shmidt, S Pradhan, and M Lipson,"Micrometre-scale silicon electro-optic modulator" Nature, 435, 325-327, 2005.

[35] F Y Gardes, G T Reed, N Emerson, and C E Png, “A sub micron depletion device, photonic modulator in silicon on insulator”, Opt. Express, 13, 8854-8854,, 2005.

[36] C A Barrios and M Lipson, "Modeling and analysis of high-speed electro-optic modulation in high confinement silicon waveguides using metal-oxide-semiconductor configuration," $J$. Appl. Phys., 96, 6008-6015, 2004.

[37] G T Reed and C E Png, "Silicon optical modulators", Materials Today, $\underline{\text {, }, ~ 40-50, ~} 2005$. 


\section{FIGURE CAPTIONS}

Figure 1a The singlemode condition and the zero birefringence condition for a rib waveguide with height $\mathrm{H}=1.35 \mu \mathrm{m}$.

Figure $1 \mathrm{~b}$ The singlemode condition and the zero birefringence condition for a rib waveguide with height $\mathrm{H}=1.5 \mu \mathrm{m}$.

Figure 2a Modelling of a directional coupler to show polarisation independent transfer of power from the left waveguide to the right waveguide, for a coupling length of $500 \mu \mathrm{m}$.

Figure $2 \mathrm{~b}$ Measured spectral response of a polarisation independent racetrack resonator.

Figure 3a Modelled response of cascaded ring resonators based upon experimentally measured devices with bend radii of $25 \mu \mathrm{m}$ and $50 \mu \mathrm{m}$, a coupler length of $500 \mu \mathrm{m}$, and waveguide height of $1.35 \mu \mathrm{m}$.

Figure $3 \mathrm{~b}$ Modelled response of serially coupled double-ring resonators based upon experimentally measured devices with bend radii of $25 \mu \mathrm{m}$ and $50 \mu \mathrm{m}$, a coupler length of $210 \mu \mathrm{m}$, and waveguide height of $1.35 \mu \mathrm{m}$. 
Figure 4a Measured FWHMs of a Triple Ring Resonator (TRR), a Double Ring Resonator (DRR) and a Single Ring Resonator (SRR) with similar serially coupled rings are 17, 22 and $27 \mathrm{pm}$, respectively.

Figure 4b Theoretical and measured responses of the Double Ring Resonator (DRR) with similar rings.

Figure 5a Cross section of a castellated surface grating coupler fabricated on a $1 \mu \mathrm{m}$ silicon waveguide. The period is approximately $400 \mathrm{~nm}$, designed to operate at $\lambda=1.3 \mu \mathrm{m}$, and the output efficiency was $72 \%$.

Figure $5 \mathrm{~b}$ Cross section of a blazed surface grating coupler fabricated on a $1 \mu \mathrm{m}$ silicon waveguide. The period is approximately $383 \mathrm{~nm}$, designed to operate at $\lambda=1.3 \mu \mathrm{m}$, and the output efficiency was $84 \%$.

Figure 6 Simulation of $30 \mathrm{keV}$ Oxygen ions implanted into the surface of a silicon waveguide, showing the implanted profile and ion induced damage prior to annealing.

Figure 7a Cross sectional view of a Dual Grating Assisted Directional Coupler (DGADC), to couple light from an optical fibre to a small silicon waveguide

Figure $7 \mathrm{~b}$ Predicted efficiency of the DGADC depicted in figure $7 \mathrm{a}$ for a range of combinations of grating heights 
Figure. 8a Cross sectional view of a p-i-n phase modulator, designed for operation in a $0.98 \mu \mathrm{m}$ height rib waveguide

Figure. $8 \mathrm{~b}$ Cross sectional view of a depletion modulator for inclusion in a push-pull Mach Zehnder interferometer.

Table 1. A comparison of the FSR achieved for ring resonators fabricated with varying circumference. 
Figure 1(a)

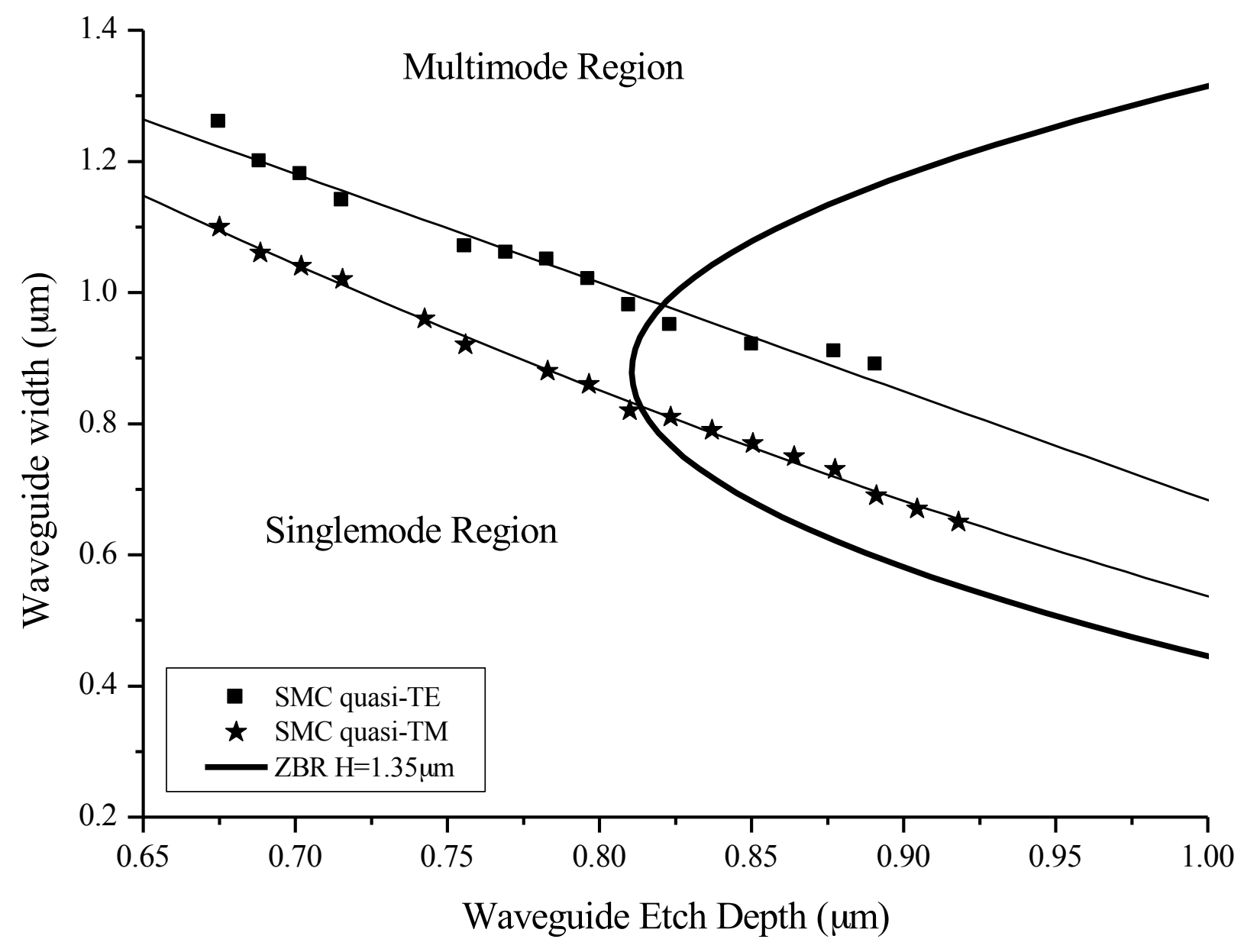


Figure 1(b)

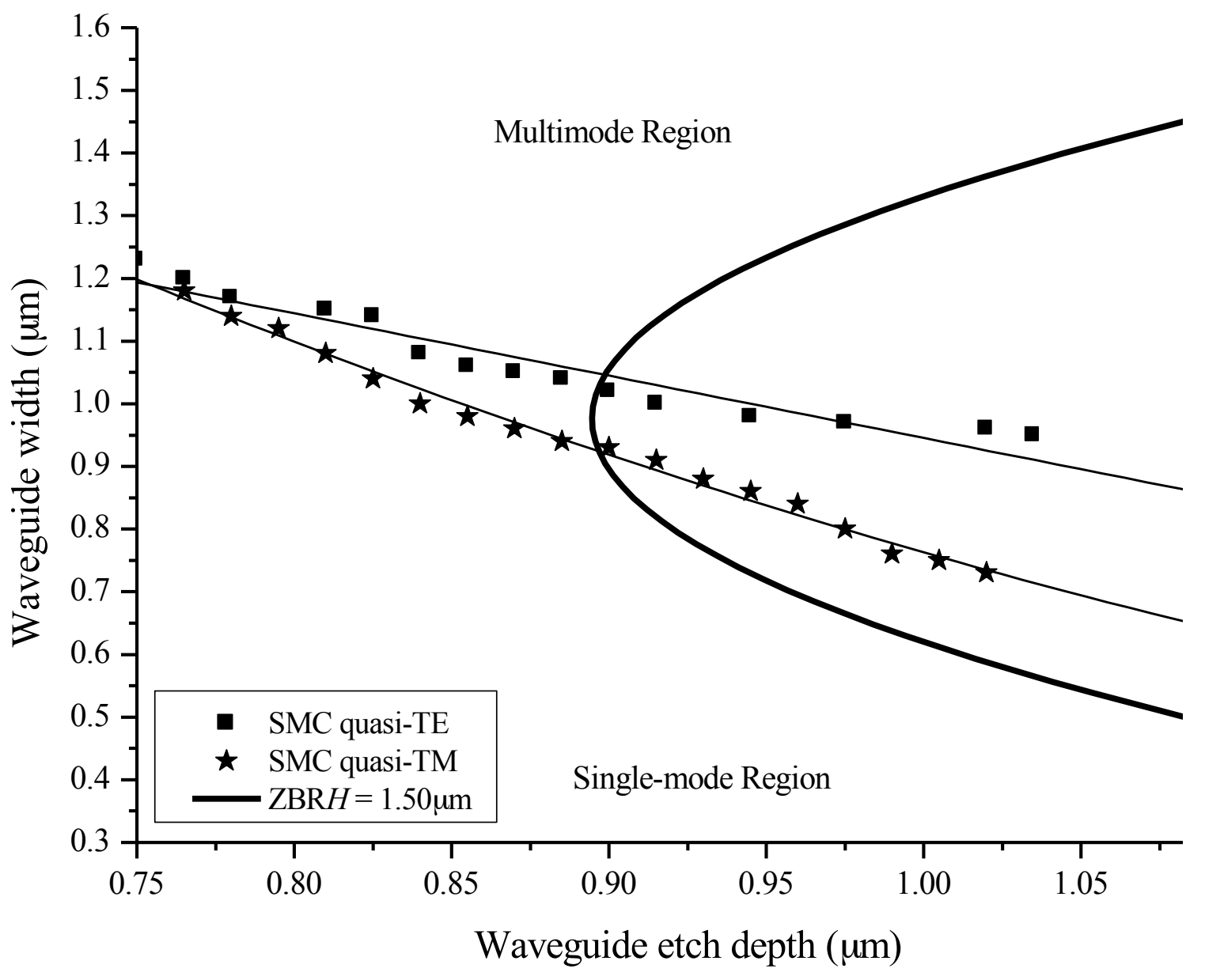


Figure 2(a)

TE

TM

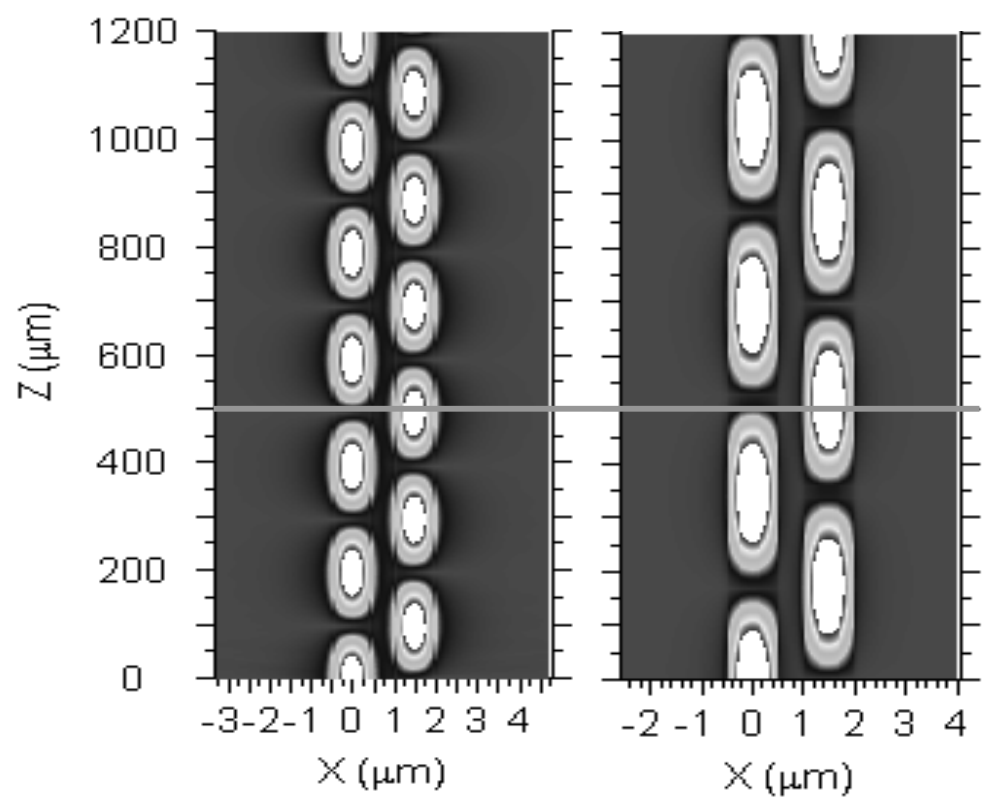

(b) 
Figure 2(b)

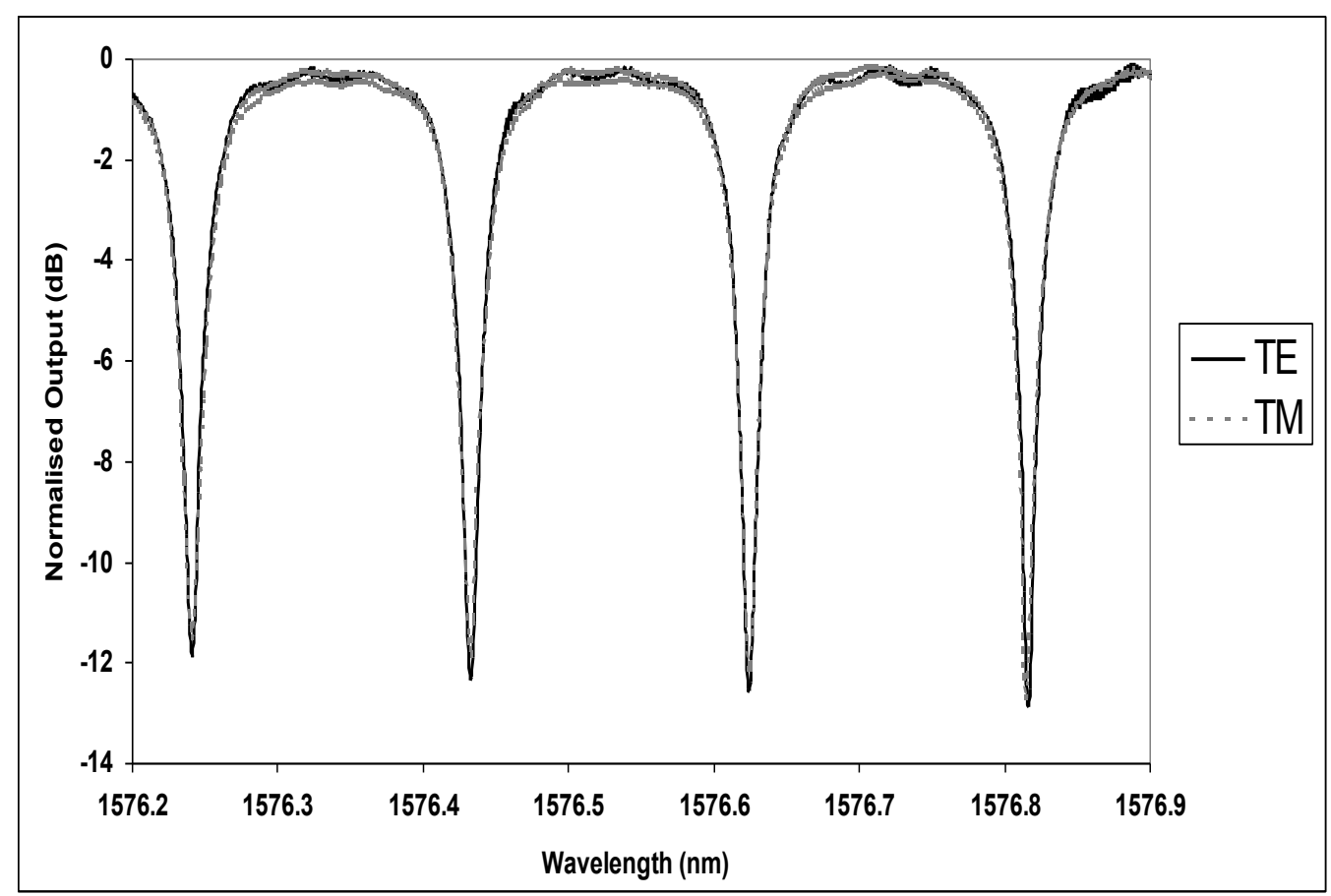


Figure 3a

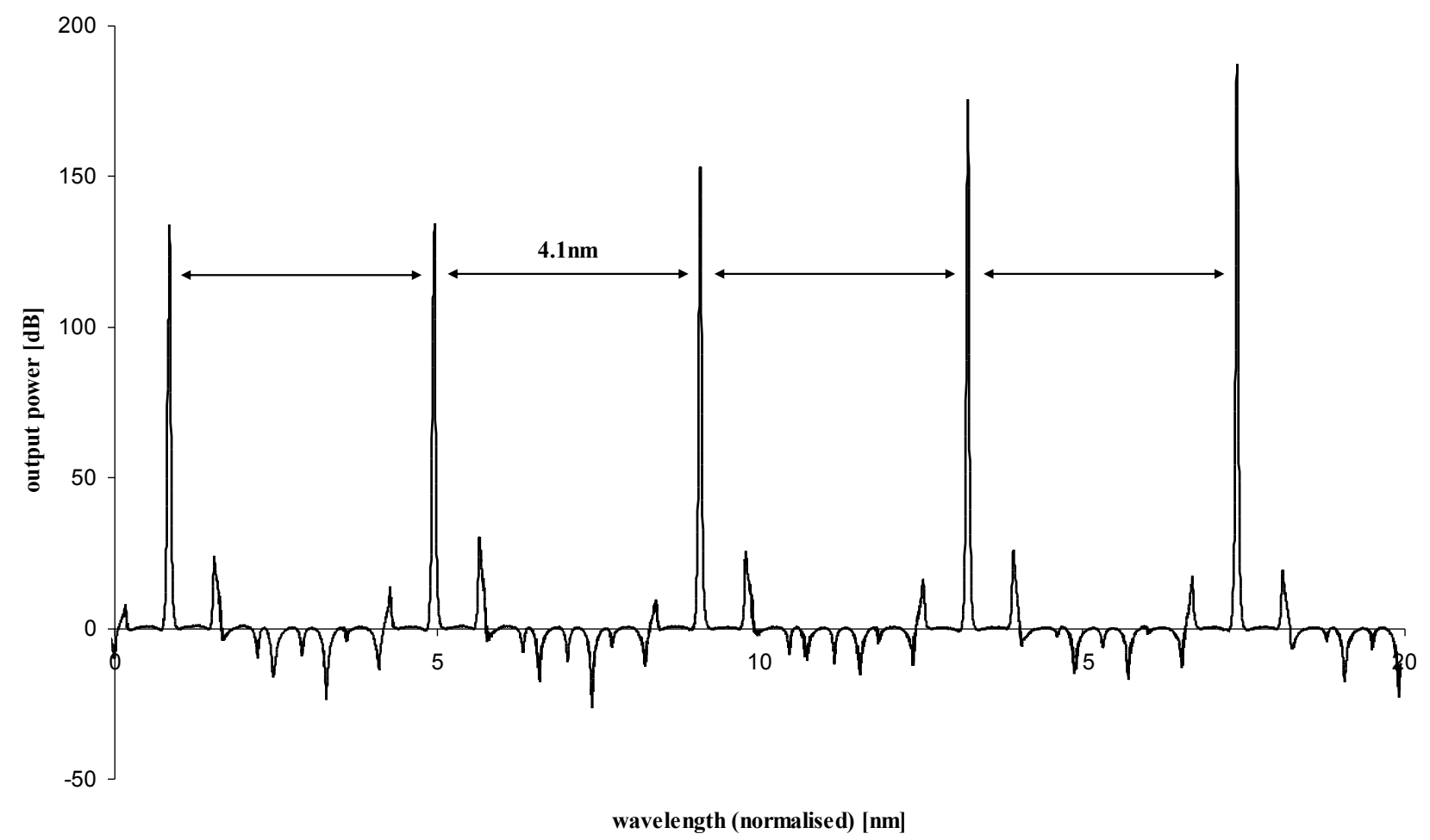


Figure 3(b)

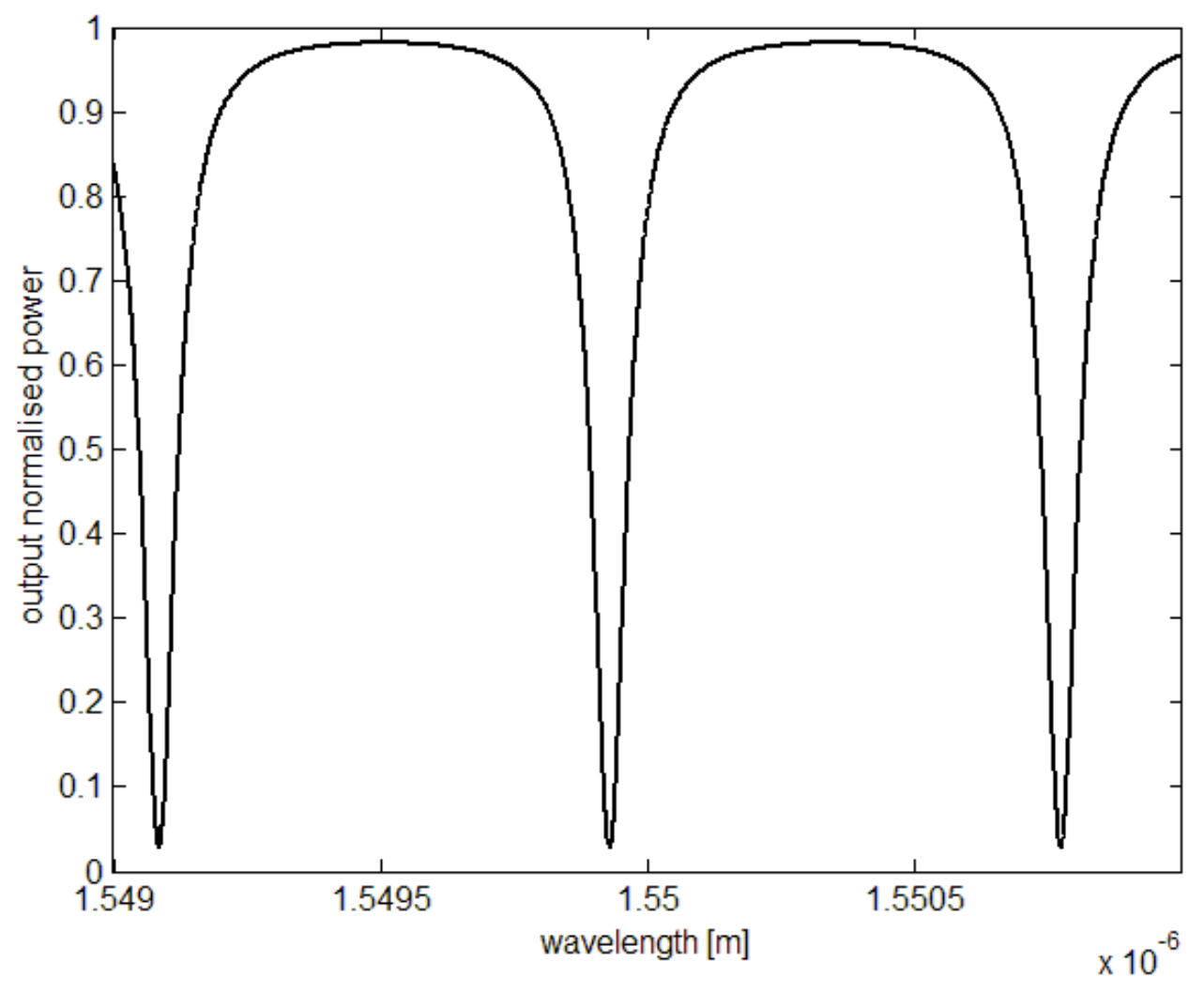


Figure $4 \mathrm{a}$

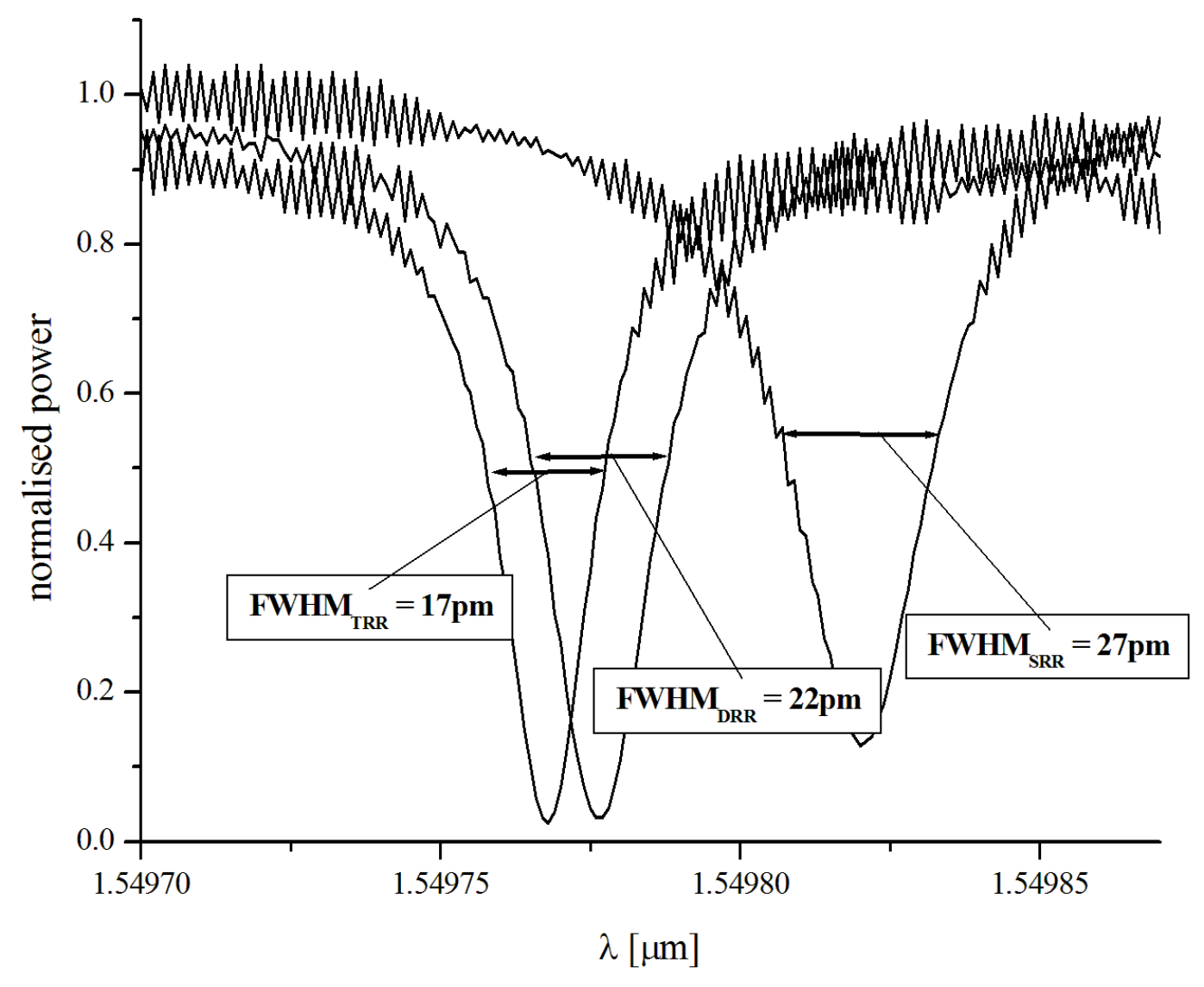


Figure $4 \mathrm{~b}$

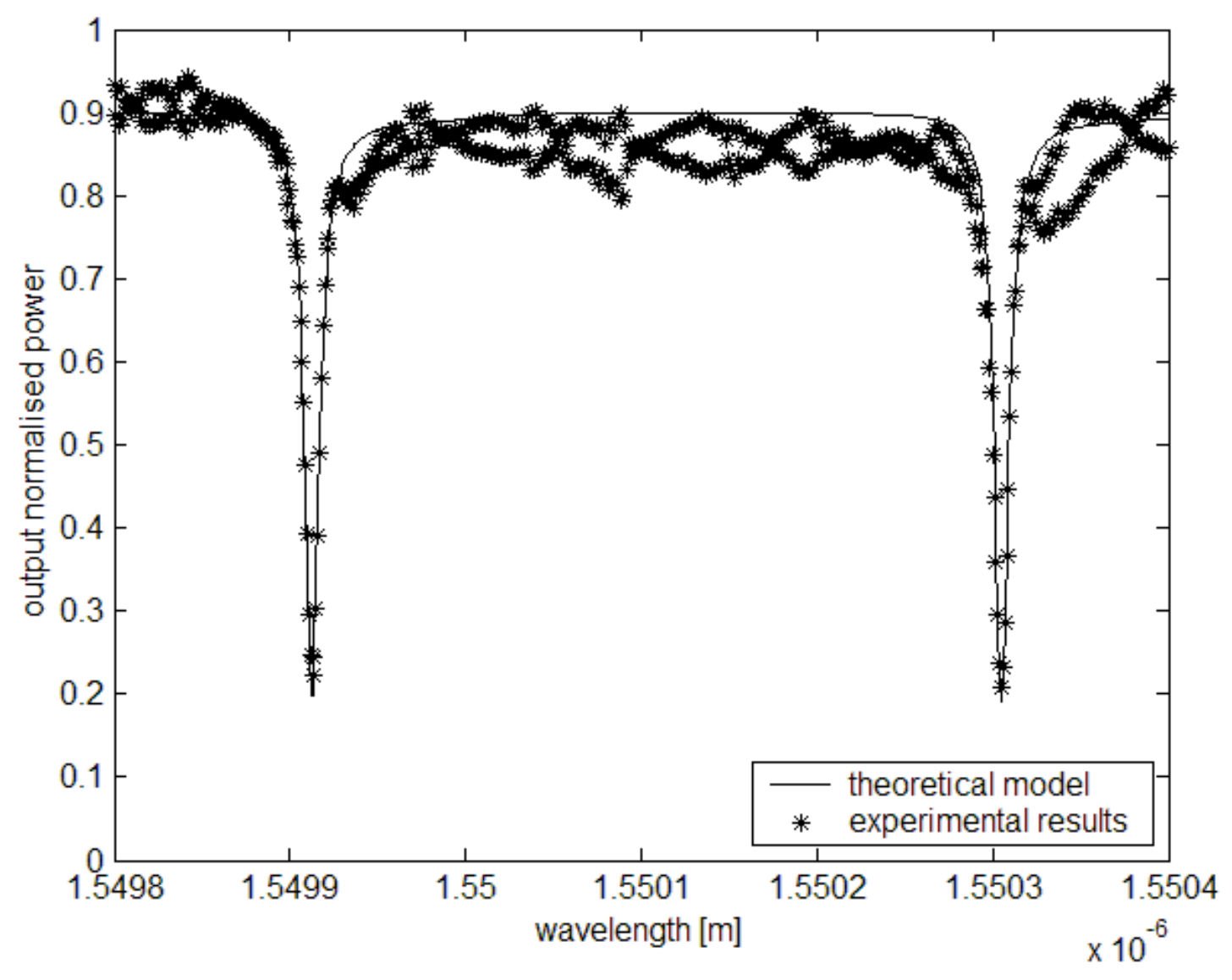


Figure 5a

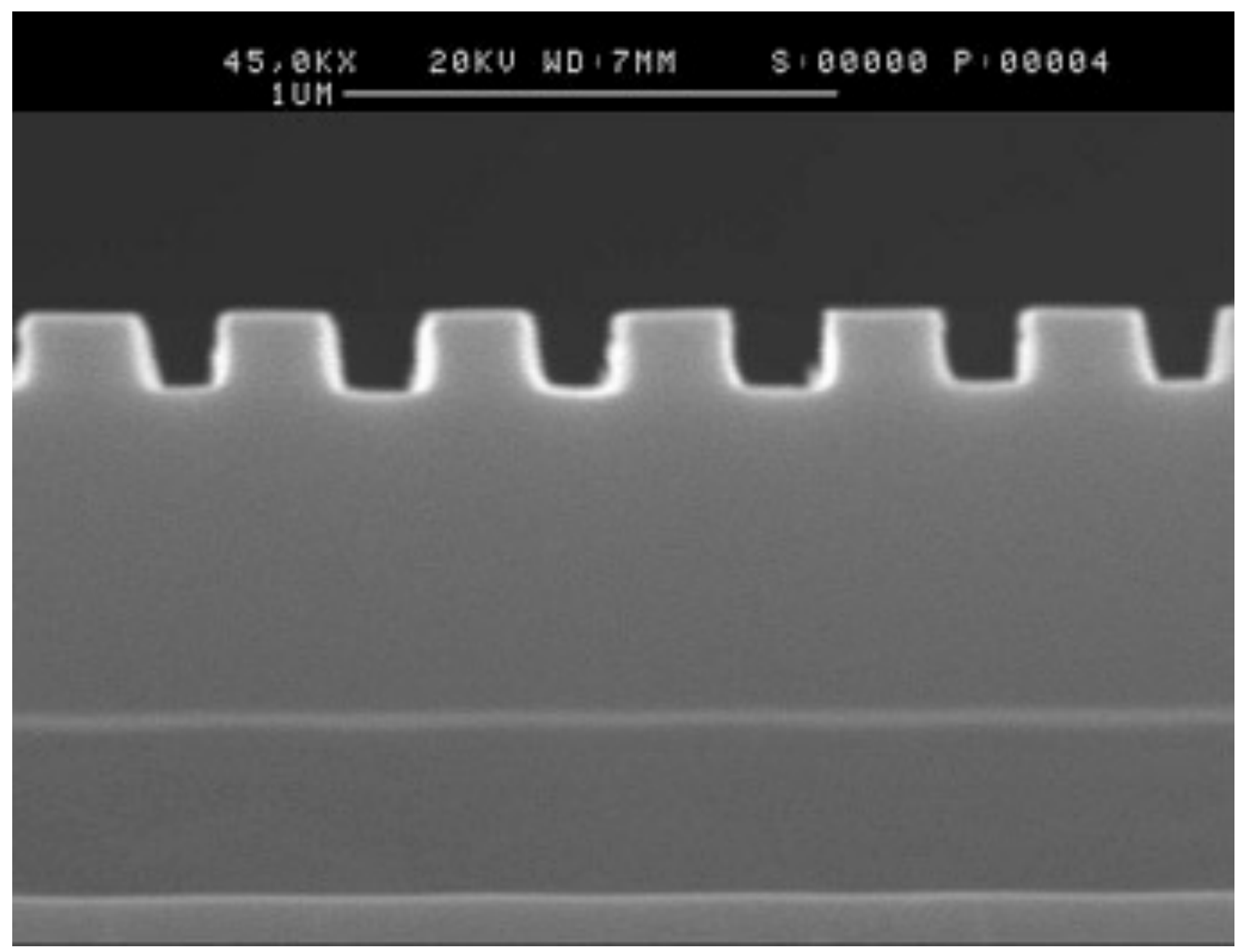


Figure $5 b$

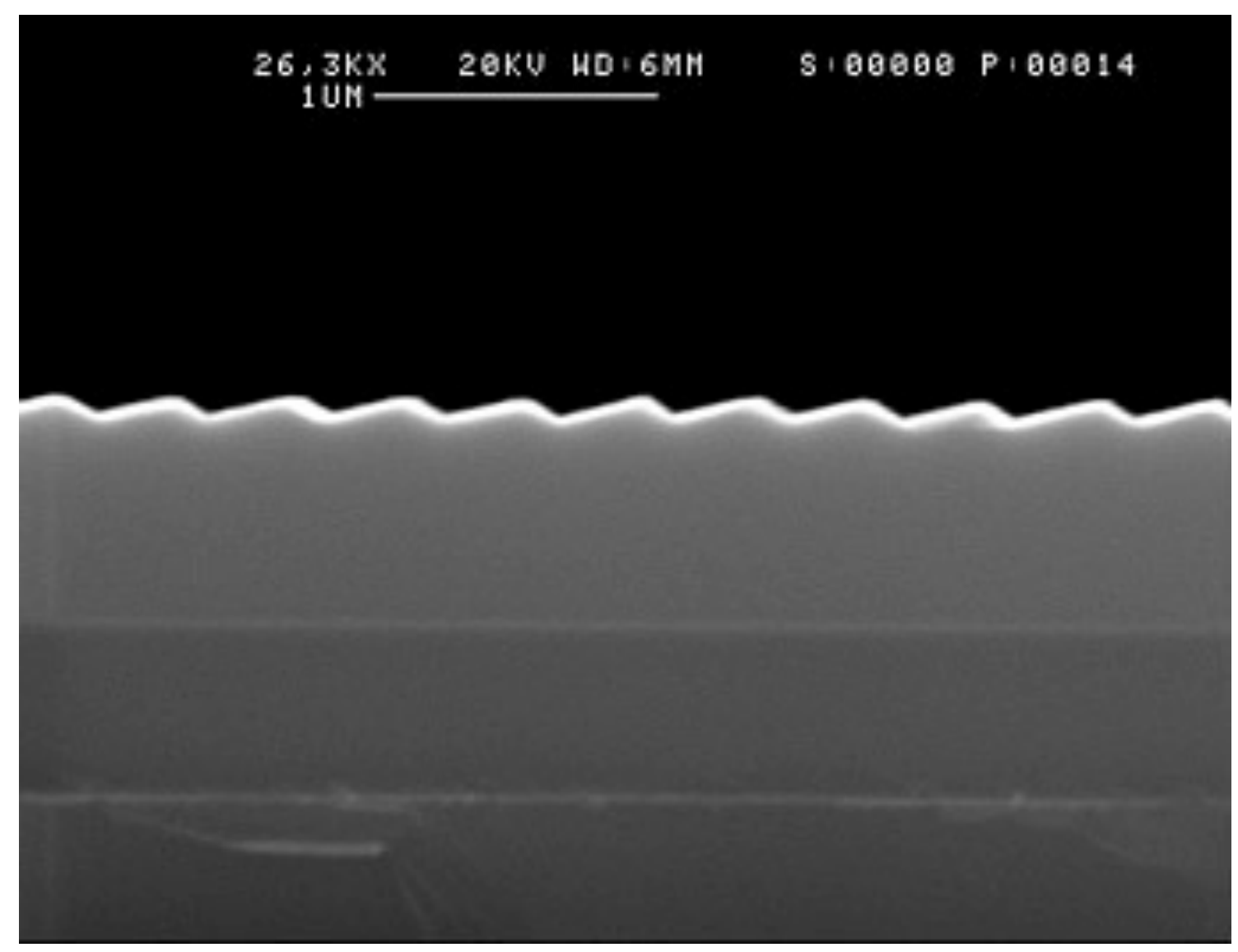


Figure 6

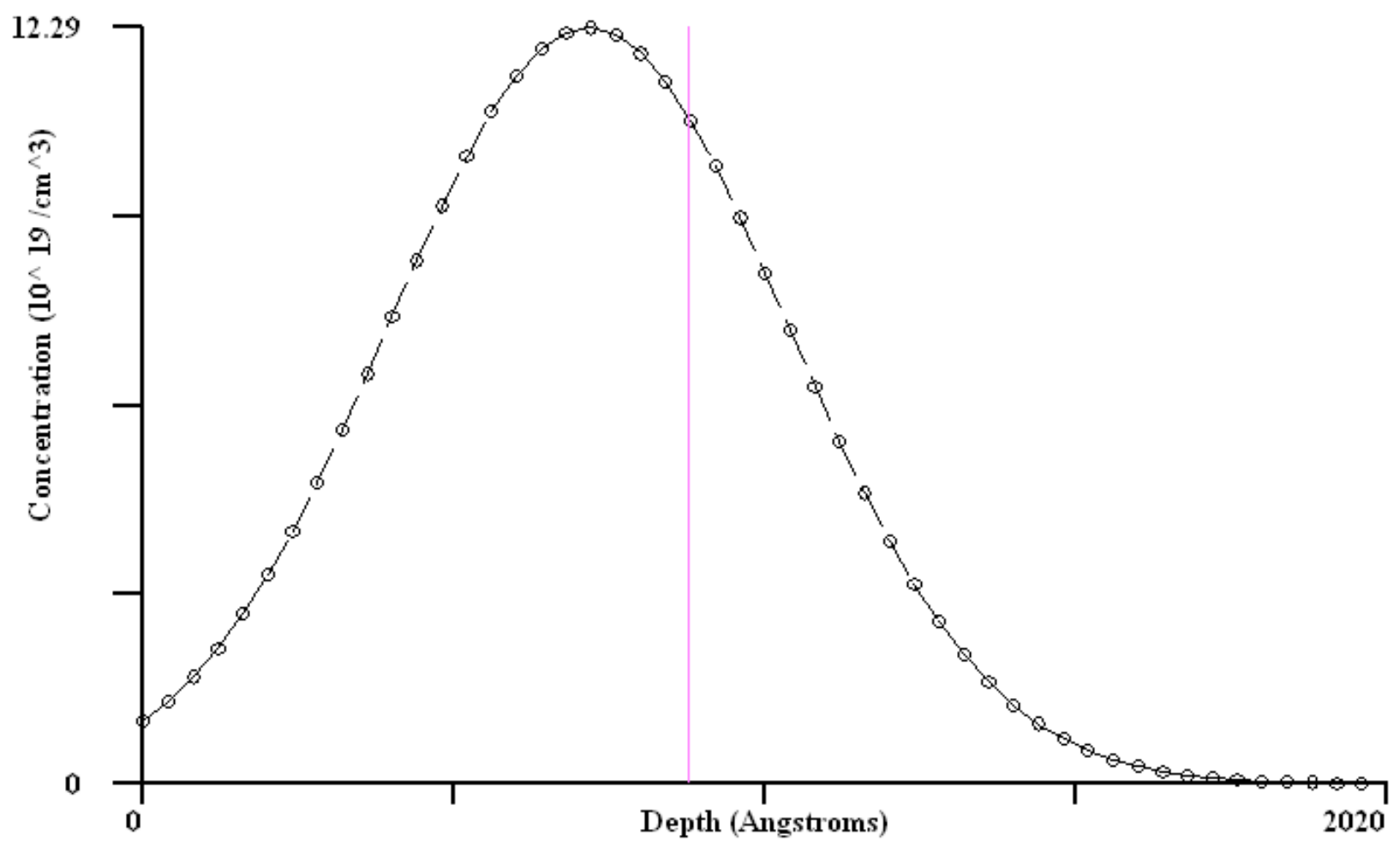


Figure $7 \mathrm{a}$

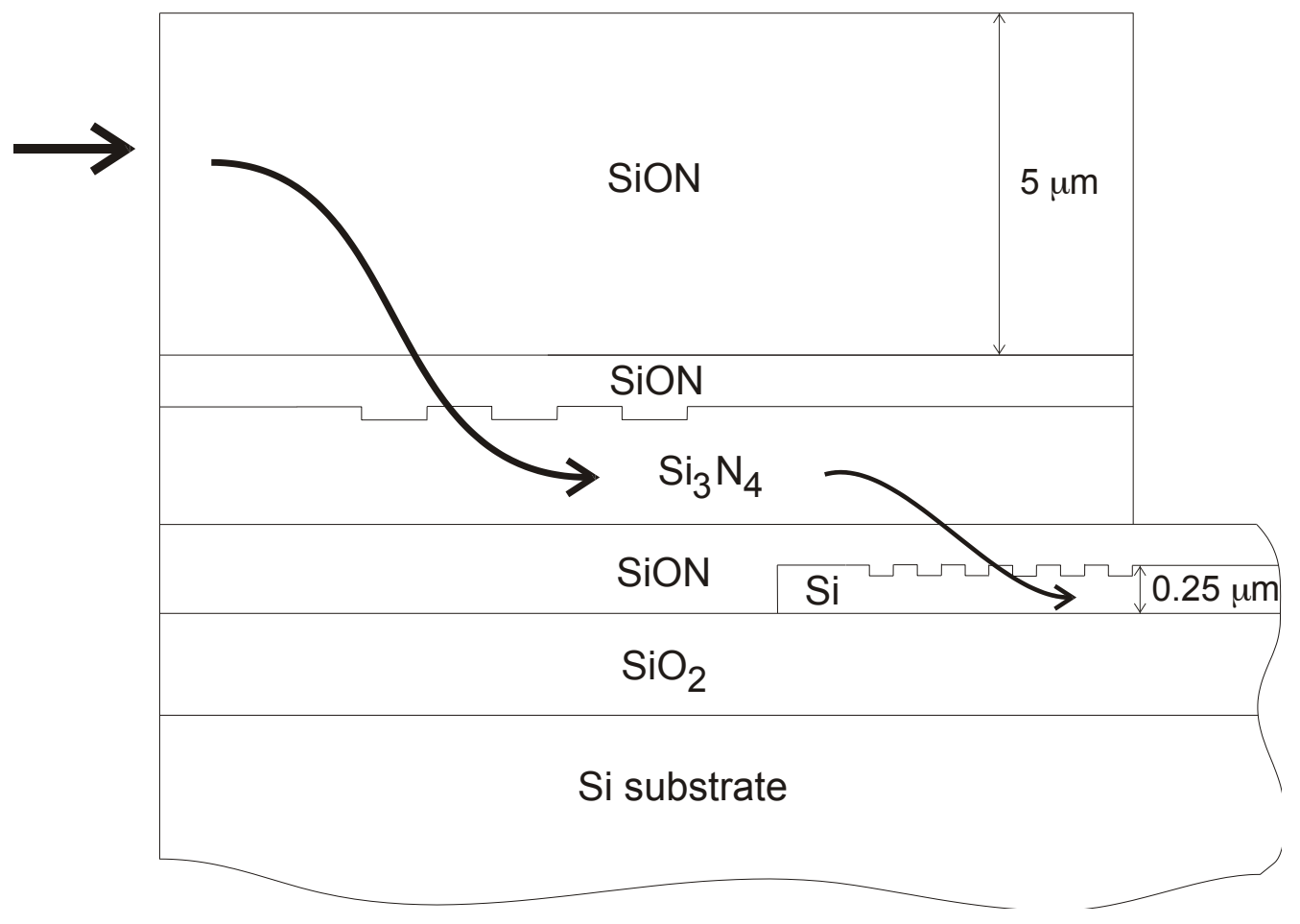


Figure $7 b$

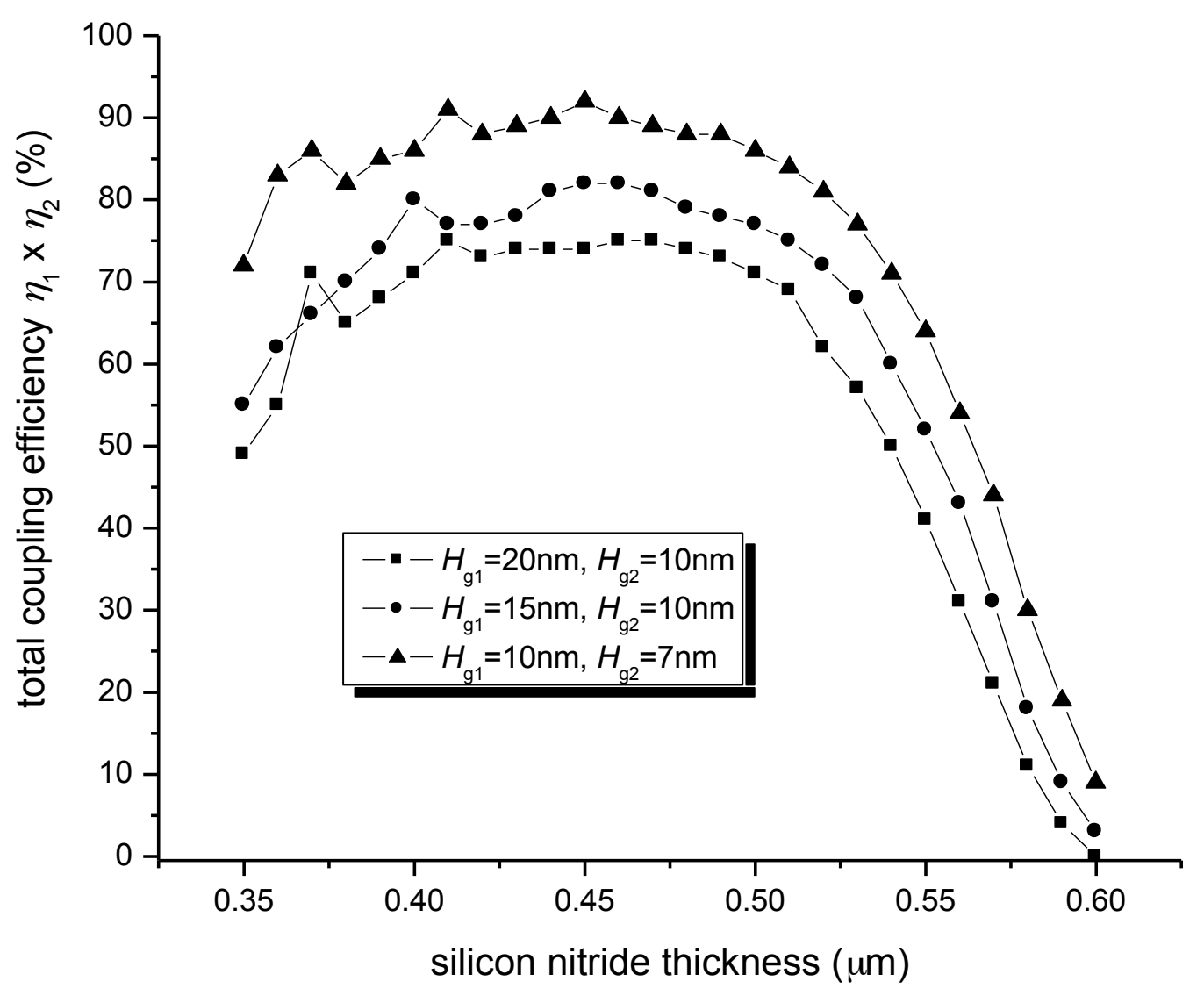


Figure 8a

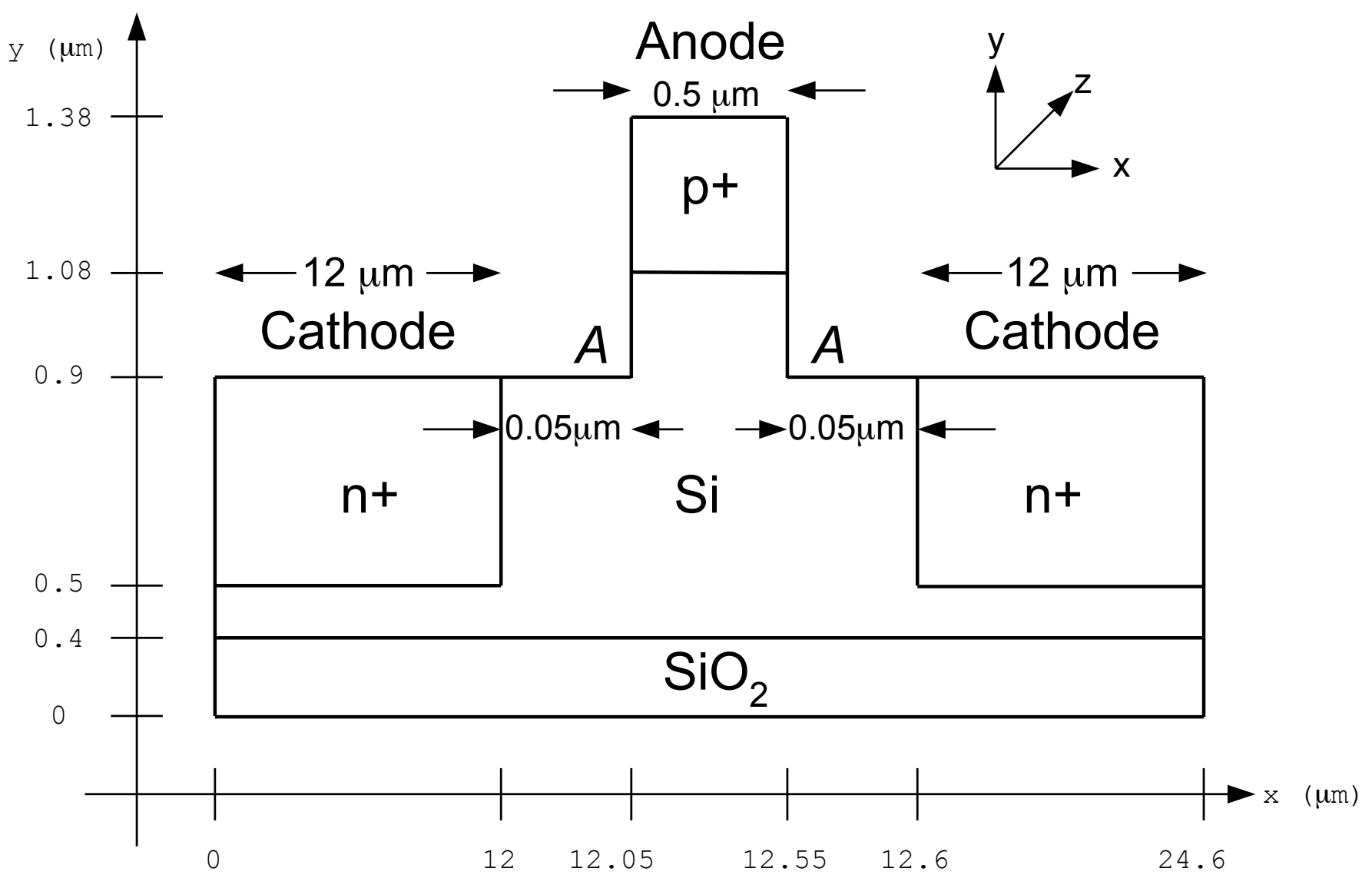


Figure $8 b$

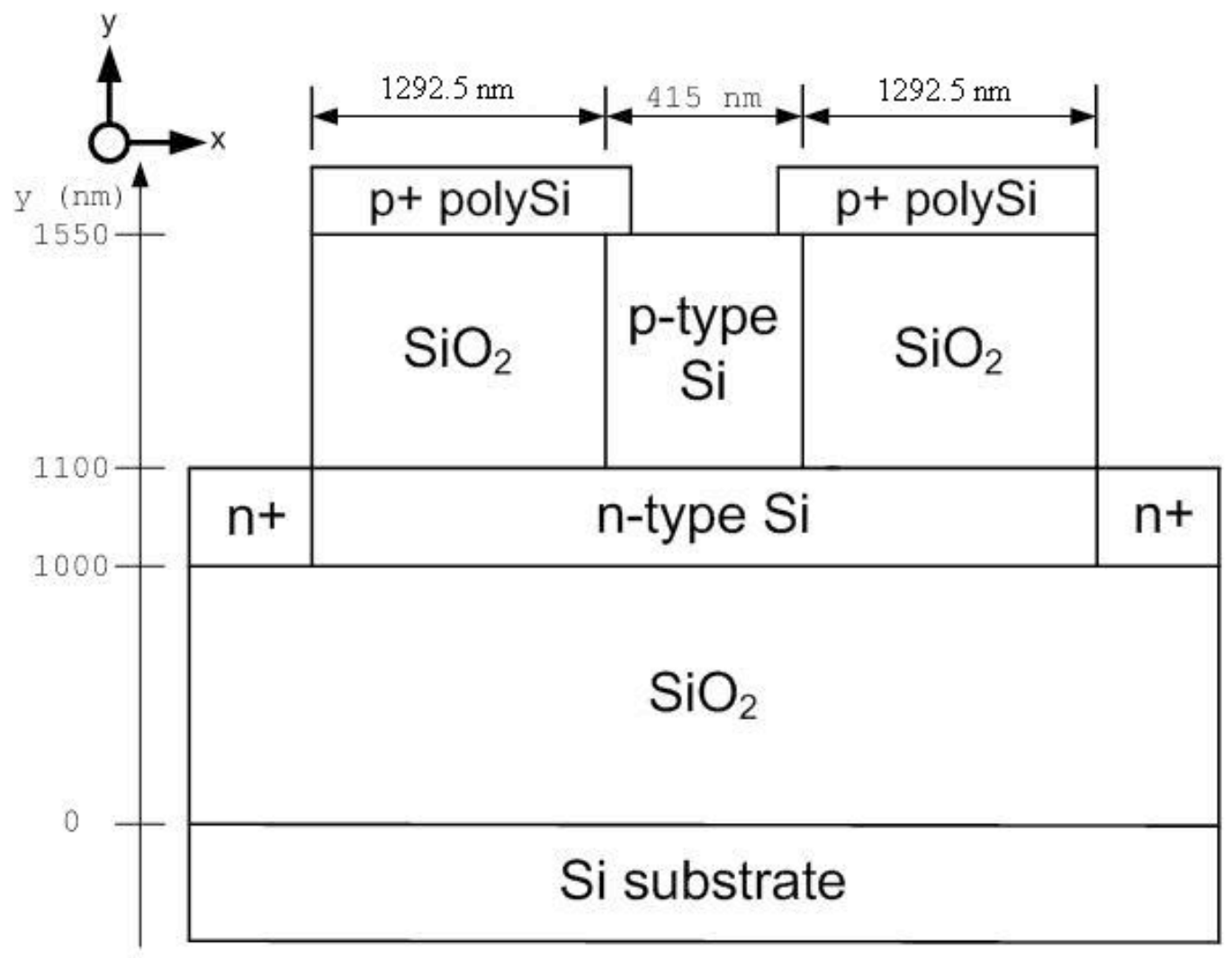


Table 1

\begin{tabular}{|l|l|l|l|}
\hline Bend Radius & Total circumference & FSR & Q \\
\hline $400 \mu \mathrm{m}$ & $3513 \mu \mathrm{m}$ & $0.193 \mathrm{~nm}$ & 90,000 \\
\hline $300 \mu \mathrm{m}$ & $2505 \mu \mathrm{m}$ & $0.251 \mathrm{~nm}$ & 170,000 \\
\hline $200 \mu \mathrm{m}$ & $1877 \mu \mathrm{m}$ & $0.333 \mathrm{~nm}$ & 30,000 \\
\hline $50 \mu \mathrm{m}$ & & & 30,000 \\
\hline $25 \mu \mathrm{m}$ & $934 \mu \mathrm{m}$ & $0.691 \mathrm{~nm}$ & \\
\hline
\end{tabular}




\section{AUTHOR BIOGRAPHIES}

Graham T Reed joined the University of Surrey in 1989 with the aim of establishing a research activity in guided wave optoelectronics, and now leads an internationally recognised group. He is responsible for initiating a new research field in the UK on Silicon Integrated Optical Circuits, and his group has produced a series of leading technical advances in the field worldwide, notably in optical modulators, grating couplers, and optical sensing applications. A testament to the originality and potential of the silicon work is that Bookham Technology plc adopted it as their core business in the early 1990s. Professor Reed's work is built upon collaborative arrangements with both industry and academia alike. His work has been associated with companies and universities in the UK, the USA, France, Italy, Germany, Japan and Singapore. Professor Reed has contributed to more than 150 publications in the field of guided wave optoelectronics, coauthored the first text book on silicon photonics, and contributed to several patents. He is a Fellow of the IEE.

Goran Z Mashanovich obtained his Dipl. Ing. and M.Sc. degrees in Electrical Engineering from the Faculty of Electrical Engineering, University of Belgrade, Serbia, and $\mathrm{PhD}$ from the University of Surrey, UK. After working for 5 years as a Teaching and Research Assistant at the Faculty of Electrical Engineering, University of Belgrade, he joined the Silicon Photonics Group at the School of Electronics and Physical Sciences, University of Surrey, in 2000. His research interests include silicon photonics and fibre optic sensors.

William R Headley received his Ph.D. in Electronics Engineering in 2005 from Surrey University, Guildford, UK. He received his B.Sc. and M.Sc. in Physics from the Purdue 
University, Lafayette, IN, USA in 1997 and 2001 respectively. During his Ph.D. work he investigated the polarisation independence of optical ring resonators using rib waveguides. His research interests include integrated photonics for telecommunications devices, polarisation effects in waveguides, and optical telecommunications networks in general.

Branislav Timotijevic was born in Serbia in 1978. He received B.Sc.E.E. degree in 2002 from Faculty of Electrical Engineering, University of Belgrade. In April 2004, he was awarded an Overseas Research Scholarship (ORS) Award and sponsorship from the Intel Corporation to pursue his Ph.D. studies in the area of silicon-based integrated optics under the supervision of Professor G.T. Reed. His current research interests include optical filters in silicon-on-insulator and silicon photonics.

Frederic Y Gardes was born in France in 1977. He received a DUT in Physics Measures in 1999 from the IUT of Lannion, a Bsc degree (Honours) in 2001 from the University of Portsmouth and a Msc in Optoelectronics in 2002 from the University of Northumbria. In 2004, he received a scholarship from EPSRC to pursue his Ph.D. degree under the supervision of Professor Reed. His research interests include integrated optics in silicon-on-insulator (SOI), numerical simulation of optical and semiconductor components, optical modulators based on the plasma dispersion effect and ring resonators.

Seong Phun Chan was born in Malaysia in 1976. He received B.Eng degree (Honours) in 2000, and his PhD in 2005 from the School of Electronics \& Physical Sciences, University of Surrey. In 2001, he received a scholarship from Agilent Technologies (Singapore) to pursue his Ph.D. 
degree under the supervision of Professor Reed. His research interests include integrated optics in silicon-on-insulator (SOI), numerical simulation of optical components; waveguide based Bragg grating devices and thermo-optic phenomena in SOI waveguide.

Peter Waugh was born in Kingston, Surrey, England in 1952 and gained a degree in Electronics at Surrey University in 1976 and since then has been mainly employed as an Electronic engineer. In 2003 he retired and joined Professor Reed's group to study for a PhD in Silicon Photonics adding to a Diploma in Electronics and a Master's Degree in Optical Electronics previously taken and pursuant on a lifetime's interest in Optical Electronics.

Neil G Emerson is a Senior Lecturer in the Department of Electronic Engineering at the University of Surrey and has been a member of the Surrey Centre for Research in Ion Beam Applications (SCRIBA) since joining the University in 1984. Dr. Emerson was formerly a research fellow with the Edinburgh Microfabrication Facility at the University of Edinburgh where he was responsible for IC wafer process measurement and testing. He has a broad background in semiconductor measurement and instrumentation and has published many papers on a range of topics in the silicon/compound semiconductor field. Specific interests have been in the areas of ion beam synthesis of low resistivity layers in amorphous silicon and in studies of electrically active defects and mechanical strain in $\mathrm{Ge}^{+}$implanted silicon. His recent research has involved the production of ultra-shallow layers in silicon, this being part of a broader programme aimed at developing technology for producing future generation, sub-100nm CMOS; Spin-offs from this work are currently being exploited by collaborations in the field of silicon photonics. 
Ching Eng Png was born in Singapore in 1974. He received the B.Eng. in Electronic and Electrical Engineering (First Class Honours) and the Ph.D. degrees from the University of Surrey, U.K., in 1999 and 2004 respectively. His Ph.D. work was supervised by Prof. G. T. Reed and focused on silicon photonics. From November 1999 to September 2000, he was with Agilent Technologies, Singapore, as a Gigabit Optical Transceiver Engineer. He is currently with the Institute of High Performance Computing, A*Star, Singapore, working on bio-photonics and silicon photonics. Dr. Png was awarded the Royal Academy of Engineering Prize and the Institution of Electrical Engineers (IEE) Hudswell International Research Scholarship for his work on silicon optical modulators.

Mario Paniccia is a senior member or IEEE and currently the Director of Photonics Technology Lab at Intel Corporation. Mario currently directs a research group with activities in the area of Silicon Photonics. The team is focused on developing silicon-based photonic building blocks using standard CMOS processing for future use in enterprise and data center communications. Mario has worked in many areas of optical technologies during his career at Intel including optical testing for leading edge microprocessors, optical communications and optical interconnects. His teams pioneering activities in silicon photonics have led to first silicon modulator with bandwidth $>1 \mathrm{GHz}$ (2004) and the first continuous wave Silicon laser breakthrough (2005). He has published numerous papers, including 3 Nature papers, 2 book chapters, and has over 61 patents issued or pending. Mario earned a B.S. degree in Physics in 1988 from the State University of New York at Binghamton and a Ph.D. degree in Solid State Physics from Purdue University in 1994. 
Ansheng Liu received the Ph.D. degree from the University of Aalborg, Aalborg, Denmark, in 1992. He is currently a Research Scientist and Senior Staff Member with the Photonic Technology Laboratory, Intel Corporation, Santa Clara, CA, where he is developing silicon photonic devices in the Corporate Technology Group. Before joining Intel in 2000, he worked at NASA Ames Research Center, the National Institute of Standards and Technology, and at the University of Aalborg as an assistant professor. His interests include nonlinear optics of nanostructures, near-field optics, opto-electronics, and photonics.

Dani Hak was born in Israel in 1975. He received B.Sc in computer sciences and physics from the Hebrew University in Jeruslaem at 2003. Since 2002 he worked for Intel Corporation in process development of Silicon photonics devices. Currently he is studying for M.Sc.E.E in TelAviv university. His current research interests include waveguide based detectors.

Vittorio M N Passaro was born in Bari on 23 November 1962. In February 1988 he received his Laurea Degree cum laude in Electronic Engineering at University of Bari, and in July 1992 his Ph.D. in Electronic Engineering, curriculum Optoelectronics. Since 1 October 2000 he is associate professor of Electronic Technologies at Politecnico di Bari. Since 1988 his research interests are focused on several theoretical and experimental aspects of optoelectronic technologies and integrated optics. He is involved, often with original contributions, in modelling, design and simulation of a number of integrated optical devices and circuits for optical signal processing, telecommunications and sensor applications, by considering a number of materials, in particular ferroelectric, semiconductors (silicon and III/V alloy compounds) and ferromagnetic garnets. He has also been involved in dry etching and proton exchange technologies, in particular on the understanding of the physical-chemical mechanisms occurring 
during the formation of lithium niobate optical waveguides, and the relationships with their optical properties. Currently he is involved in silicon photonics technology. He is author or coauthor of more than 140 papers published in international journals and conference proceedings and 2 international patents. He is IEEE Senior Member and OSA Member. 